\title{
Novas Tecnologias em Endodontia e seu uso no atendimento de pacientes de uma unidade de saúde pública: Cinco anos de atividade extensionista
}

\section{New technologies in Endodontics and its use in the care of patients in a public health unit: four years of extensionist activity}

Thereza Cristina Ávila Berlinck ${ }^{1}$, Justine Monteiro Monnerat Tinoco ${ }^{2}$, Cláudio Malizia Alves Ferreira ${ }^{3}$, Milena do Valle Tomaz ${ }^{4}$, Paulo Rogério Nunes Barbosa ${ }^{5}$, Luciana Moura Sassone ${ }^{6}$

\footnotetext{
1 Professora. Universidade do Estado do Rio de Janeiro (UERJ), Brasil. E-mail: therezaberlinck@gmail.com

2 Professora. Universidade Federal do Rio de Janeiro (UFRJ), Brasil. E-mail: justinemonnerat@yahoo.com.br

3 Professor. Universidade do Estado do Rio de Janeiro (UERJ), Brasil. E-mail: cla_ferreira@yahoo.com.br

4 Acadêmica. Universidade do Estado do Rio de Janeiro (UERJ), Brasil. E-mail: milenavtomaz@gmail.com

5 Residente. Escola Nacional de Saúde Pública Sérgio Arouca (ENSP - FIOCRUZ), Brasil. E-mail: paulo96nunes@hotmail.com

6 Professora. Universidade do Estado do Rio de Janeiro (UERJ), Brasil. E-mail: lucianasassone@gmail.com
}

Recebido em: 24/07/2019 | Aprovado em: 20/02/2020

DOI: $10.12957 /$ interag.2019.53544

\section{Resumo}

A Extensão Universitária é uma importante ferramenta de interação entre o estudante universitário e a comunidade, sendo ambos beneficiados. $O$ projeto de extensão "Tratamento endodôntico simplificado visando sua aplicação no SUS" tem como objetivo somar forças junto ao SUS no atendimento à comunidade, de forma a reduzir a demanda reprimida e ao mesmo tempo proporcionar aos pacientes tratamentos endodônticos com novas tecnologias. Este projeto tem contribuído para a formação de recursos humanos, para a produção de conhecimento científico e para a prestação de um tratamento com maior eficiência para a comunidade. Por meio de resultados parciais da pesquisa desenvolvida no projeto, foi possível verificar que a inclusão da instrumentação acionada a motor e outras tecnologias durante o tratamento endodôntico, permitiu redução no tempo de atendimento e consequentemente contribui para a diminuição da demanda reprimida de pacientes encaminhados para o setor de Endodontia.

Palavras-chave: Odontologia. Extensão. Saúde. SUS. Endodontia

Área temática: Ciências da Saúde. Linha de extensão: Saúde Humana.

\begin{abstract}
The University Extension is an important tool for the interaction between student and the community, in which all have great benefit. The extension project "Simplified endodontic care for its application in the SUS" aims to help the SUS on attending the community in order to expedite endodontic treatment and reduce demand with the help of new technologies. This project contributed to human resources formation, to product scientific knowledge and to the provision of a treatment with the highest efficiency for the community. Partial research results of this project, show that technology in endodontics may reduce the time of treatment and consequently increased the number of attendance of patients referred to endodontic clinic.
\end{abstract}

Keywords: Dentistry. Extention. Health. SUS Endodontics 


\section{Introdução}

A Universidade está fundamentada sob três pilares: ensino, pesquisa e extensão. ${ }^{1} \mathrm{~A}$ Extensão Universitária é uma das formas da Universidade devolver à sociedade onde está inserida, os investimentos feitos durante a formação acadêmica. Isso é realizado pela transmissão de conhecimento, aplicação de novas tecnologias desenvolvidas e a prestação de serviço. ${ }^{1}$ Além disso, a Extensão Universitária contribui para a formação do estudante universitário por meio de atividades acadêmicas de ensino e pesquisa com atendimentos à comunidade, permitindo que o estudante se desenvolva profissionalmente enquanto exerce sua cidadania ${ }^{2,3}$

$\mathrm{Na}$ área da saúde, os projetos de extensão muitas vezes se alinham com a prática clínica do Sistema Único de Saúde (SUS) seja na Atenção Primária em Saúde (APS), como também na realização de atendimentos especializados . ${ }^{4}$ A Atenção Primária em Saúde (APS) é uma rede de serviços, baseada em todos os princípios do SUS (universalização, equidade e integralidade), capaz de resolver $85 \%$ dos problemas da população além de garantir fluxos de referência e contra referência aos serviços especializados, de apoio diagnóstico e terapêutico, ambulatorial e hospitalar quando necessário. ${ }^{5,6}$

Para a qualificação e ampliação da assistência pública odontológica de média complexidade foram instituídos os Centros de Especialidades Odontológicas (CEO), que devem atender à demanda de procedimentos especializados e realizar a contra referência dos pacientes para a APS concluir os tratamentos. ${ }^{4}$ Os CEOs são as unidades de referência para a realização de atendimentos nas áreas de endodontia, periodontia, cirurgia buco-maxilo-facial, próteses e atendimento para portadores de necessidades especiais. ${ }^{?}$

Uma das especialidades que possui grande demanda nos CEOs é a Endodontia. Porém, alguns fatores como o número insuficiente de profissionais especializados, o longo tempo de espera e o número de consultas para finalização do tratamento endodôntico vêm contribuindo para o aumento da demanda reprimida. ${ }^{8,94}$ Da mesma forma, o elevado percentual de evasão dos pacientes $(57,93 \%)$, durante o tratamento endodôntico, também preocupa as autoridades responsáveis que se propõem a garantir acesso universal e integral a saúde bucal da população. ${ }^{10} \mathrm{~A}$ necessidade de aumentar a demanda dos atendimentos odontológicos vem estimulando a criação 
de estratégias que possam evitar a perda do elemento dentário, assim como também diversas consequências no sistema estomatognático. ${ }^{11,12,14}$

Neste contexto, surgiu à intenção de se criar um projeto de extensão na área de Endodontia que somasse forças junto ao SUS no atendimento à comunidade, de forma a reduzir a demanda reprimida e ao mesmo tempo proporcionar aos pacientes tratamentos endodônticos com as mais novas tecnologias implementadas na Endodontia.

O projeto de extensão "Tratamento endodôntico simplificado visando sua aplicação no SUS” foi iniciado, em 2014, com o objetivo de possibilitar a oferta de tratamento endodôntico na Policlínica Piquet Carneiro (PPC), uma unidade da rede pública de saúde no Rio de Janeiro ligada a UERJ, assim como proporcionar experiência de trabalho na rede pública de saúde para discentes e docentes da disciplina de Endodontia da Faculdade de Odontologia da Universidade do Estado do Rio de Janeiro (FO-UERJ). Além disso, expandir as atividades extra-muros desenvolvidas por essa disciplina e pelo programa de pós-graduação stricto-sensu em Odontologia na área de concentração Endodontia.

O objetivo deste relato é descrever a experiência do Projeto de Extensão "Tratamento endodôntico simplificado visando sua aplicação no SUS" desde o seu início em novembro de 2014.

\section{O Projeto "Tratamento endodôntico simplificado visando sua aplicação no SUS"}

A ideia inicial do projeto surgiu em decorrência da ausência de oferta de tratamento endodôntico especializado no setor de Odontologia da PPC somado à ideia de proporcionar ao discente tanto de graduação quanto de pós-graduação uma experiência em atendimento para saúde pública. O Projeto de Extensão permitiu que os discentes se desenvolvessem profissionalmente, aplicando os conhecimentos adquiridos, e pessoalmente, desenvolvendo competências e habilidades de uma atitude ética, criando vínculos com a comunidade e reconhecendo sua importância ao longo de sua vida acadêmica. Além disso, o projeto possibilitou a expansão clínica para a realização de pesquisas científicas. 
O projeto de extensão "Tratamento endodôntico simplificado visando sua aplicação no SUS" (cadastrado no SIEXT no 4613, em 2014 e no 5466 a partir de 2018) foi criado em 2014 sob a coordenação da professora Luciana Moura Sassone. Este projeto de cunho social e científico foi aprovado pelo Comitê de Ética em Pesquisa com Seres Humanos (parecer no 1.055.927/2015) e teve como finalidade avaliar a utilização de diferentes tecnologias para a realização de tratamento endodôntico na rede pública visando prestar atendimentos mais rápidos e eficazes associando simplicidade a excelência.

O projeto conta com tecnologias modernas utilizadas na Endodontia. A instrumentação dos canais radiculares é feita, em alguns casos, com instrumentos de Níquel Titânio (NiTi) acionados a motor que conferem a flexibilidade necessária para o tratamento de canais com diferentes curvaturas de forma mais rápida e previsivel. ${ }^{13}$

As tomadas radiográficas são minimizadas devido a utilização do Localizador apical NOVAPEX® (Forum Technologies, Rishon Le-Zion, Israel). Este aparelho determina o tamanho do canal a ser instrumentado por meio do conceito da impedância. ${ }^{14}$ As radiografias periapicais realizadas para diagnóstico, registro de algumas etapas do tratamento e acompanhamento dos casos clínicos, são feitas com placas de fósforo e obtidas sem a necessidade das soluções reveladora e fixadora, uma vez que a imagem é processada de forma virtual e visualizada diretamente no computador. Essa tecnologia, além de eliminar o uso de soluções prejudiciais ao meio ambiente, confere tomadas radiográficas com menor incidência de radiação, de rápida execução e acima de tudo, permite o armazenamento digital das imagens. ${ }^{15}$

A rotina dos atendimentos aos pacientes acontece às quintas-feiras das 08:00h às 11:00h na clínica odontológica da Policlínica Piquet Carneiro (PPC) conveniada à Universidade do Estado do Rio de Janeiro (UERJ) e conta com a participação de estudantes de graduação e pósgraduação stricto-sensu (área de concentração em Endodontia) da Faculdade de Odontologia da UERJ (FO-UERJ), sob supervisão docente. Os pacientes atendidos pelo projeto são provenientes da própria Policlínica e do Sistema Nacional de Regulação (SISREG). Após a conclusão do tratamento endodôntico, os pacientes são encaminhados para os profissionais responsáveis pela reabilitação funcional e estética dos elementos dentários da Policlínica Piquet Carneiro e do SISREG 
Concomitante aos atendimentos, os acadêmicos estão realizando um estudo científico que visa avaliar a eficiência da instrumentação acionada a motor comparada à instrumentação manual. Estudos comparativos entre técnicas manuais e rotatórias indicam que os instrumentos rotatórios de níquel-titânio estão associados a um menor tempo de tratamento e menor dor pósoperatória. ${ }^{15,16}$ Uma das principais causas de dor pós-operatória é a extrusão apical. ${ }^{17} \mathrm{~A}$ quantidade de debris extruídos varia de acordo com as técnicas e instrumentos empregados no tratamento endodôntico, observando-se uma correlação direta dos níveis de inflamação dos tecidos periapicais com a quantidade de extrusão de detritos. ${ }^{18,19,20,21}$

Sendo assim, o estudo desenvolvido por esse programa de extensão pretende confrontar o tempo total de atendimento, performance dos sistemas de instrumentação utilizados, bem como o percentual de dor pós-operatória relacionada à técnica utilizada.

\section{Metodologia}

O projeto de extensão "Tratamento endodôntico simplificado visando sua aplicação no SUS” do curso de Odontologia da UERJ vem sendo desenvolvido na Policlínica Piquet Carneiro (PPC), Rio de Janeiro por meio dos atendimentos ambulatoriais. Os critérios de exclusão da pesquisa são pacientes com indicação de retratamento endodôntico ou com dentes que não possam receber tratamento restaurador direto após o tratamento endodôntico.

$\mathrm{Na}$ consulta inicial é preenchida uma ficha clínica (Figura1) contendo os dados pessoais, histórico médico e odontológico, exame clínico e anamnese endodôntica detalhando a evolução da dor, condição inicial do dente a ser tratado, diagnóstico da doença, morfologia dos canais radiculares e raízes, presença ou não de lesão perirradicular e uso prévio de medicação para alívio da dor. Além disso, no verso da ficha são realizadas anotações de dados relativos ao tratamento (odontometria, lima memória, técnica de instrumentação utilizada, cimento endodôntico utilizado na obturação, tempo de consulta, medicações prescritas, dados dos procedimentos realizados e o acompanhamento de dor pós-operatória, via contato telefônico após 24, 48 e 72 horas). 
São utilizadas no projeto três diferentes técnicas de instrumentação: uma com a utilização de instrumentos acionados manualmente e duas com instrumentos acionados a motor, sendo estes os instrumentos Race ${ }^{\circledR}$ (FKG Dentaire SA, Suiça) ou Reciproc ${ }^{\circledR}$ (VDW, Munique, Alemanha) (Figura 2) que foram divididas em três grupos. Durante a fase de cateterismo e odontometria são utilizadas em todos os grupos limas tipo K (Dentsply-Maillefer, Suiça) de série especial (números: 6, 8, 10) e número 15. No grupo do sistema Race®, três instrumentos são utilizados em movimento rotatório na sequência recomendada pelo fabricante $\left(15.06,25.04\right.$ e 30.04). No grupo do sistema Reciproc ${ }^{\circledR}$, um único instrumento é utilizado em movimento reciprocante, para instrumentar os canais radiculares conforme recomendações do fabricante. Já o grupo da técnica manual é composto por aproximadamente dez instrumentos, entre limas de $1^{\text {a }}$ e $2^{\text {a }}$ série e fresas de Gates Glidden (Dentsply-Maillefer, Suiça), número 4, 3 e 2 (Figura 3), que são utilizados no sentido coroa-ápice segundo a técnica preconizada pela Disciplina de Endodontia da Faculdade de Odontologia da FO-UERJ. O descarte dos instrumentos manuais é realizado de acordo com a presença de alterações nas espirais e o descarte das brocas de Gates Glidden de acordo com distorções na mesma. Os instrumentos de uso mecanizado (Race® e Reciproc®) são utilizados somente uma vez como preconizado pelo fabricante. Para calibração dos operadores, foi realizado um treinamento pré-clínico com todas as técnicas a serem utilizadas. Dispomos de dois motores endodônticos VDW Silver Reciproc® (VDW, Munique, Alemanha), dois localizadores apicais Novapex® (Forum Technologies, Rishon Le-Zion, Israel) (Figura 4) e de sistema digital EXPRESS® de radiografia com placa de fósforo (Kavo, Santa Catarina, Brasil) (Figura 5).

As técnicas de instrumentação propostas foram executadas de forma aleatória utilizando uma tabela de randomização (www.random.org). Os atendimentos são cronometrados e sempre que o operador precise interromper o tratamento, para realizar radiografias ou acessar o computador para consultar imagens radiográficas, o cronômetro é pausado. Ao final de cada tratamento, o tempo de consulta e o número de sessões são anotados e os pacientes são orientados a informar a intensidade de dor após 24, 48 e 72 
horas por meio de contato direto realizado pelos participantes do projeto. Uma escala de dor visual analógica (EVA) é mostrada e explicada para cada paciente ao final da consulta e depois entregue para que o mesmo possa informar a intensidade de dor pós-operatória.

O detalhamento presente nas fichas clínicas, as diferenças de tempo de atendimento entre as técnicas, seus custos e as diferenças entre os níveis de dor pósoperatória possibilitaram uma análise da efetividade de cada técnica podendo ser usado como base para a indicação da implementação de novas técnicas e tecnologias no atendimento de pacientes do setor público.

\section{As principais ações e resultados parciais do projeto compreendem:}

- Prestação de serviço à comunidade:

Por meio deste projeto de extensão, foram realizados tratamentos endodônticos em pacientes da PPC e do SISREG por cirurgiões dentistas especialistas em endodontia, capacitados e treinados, com auxílio de novas tecnologias existentes no mercado. Dessa forma, o projeto tem contribuído para a prestação de serviço gratuito e de qualidade à comunidade e a redução da demanda reprimida por atendimento nessa especialidade;

- No período compreendido entre os anos de 2014 e 2018, foram atendidos 186 pacientes e realizados 162 tratamentos endodônticos em dentes anteriores e posteriores.

- Dentre as técnicas realizadas, 61 dentes foram tratados pela técnica manual; 62 pela técnica rotatória com lima RACE®; e 39 pela técnica reciprocante. Foram tratados 41 incisivos, 50 pré-molares e 71 molares em 52 pacientes do gênero masculino e em 110 do gênero feminino.

- Contribuição científica:

As diferenças entre as técnicas de instrumentação utilizadas nos atendimentos foram analisadas. 
Através dos resultados parciais desta pesquisa foi possível observar que o tempo de atendimento da técnica mecanizada foi consideravelmente menor que a manual, permitindo maior agilidade na resolução dos casos, porém não foi observado diferença no número final de consultas. Esses achados estão em conformidade com estudos comparativos prévios entre as técnicas. ${ }^{22,23}$

Além disso, o uso de diferentes técnicas de instrumentação não proporcionou diferença na intensidade de dor pós-operatória. Apesar de estudos comparativos entre técnicas rotatórias e manuais apresentarem resultados contraditórios ${ }^{21,16}$, os resultados parciais semelhantes de sensibilidade pós-operatória dessa pesquisa não contraindicam o uso de nenhuma das técnicas utilizadas.

- Contribuição para a formação de recursos humanos:

Os discentes envolvidos tiveram a oportunidade de experimentar tecnologias e recursos que não estão disponíveis na clínica de graduação e de experimentar uma verdadeira integração entre graduação e pós-graduação, garantindo mais conhecimentos e habilidades importantes na futura prática profissional tanto clínica quanto didático-pedagógica. Fizeram parte deste projeto até o presente momento 12 mestrandos, quatro doutorandos, e 15 estudantes unversitários. Os resultados e as atividades clínicas em si ainda foram utilizados para produção de material didático. Os dados e ações do projeto também foram divulgados em diversas edições da MOSTRA DE EXTENSÃO realizada durante o evento UERJ SEM MUROS.

- Redução de riscos ocupacionais ao operador:

As tecnologias utilizadas no projeto de extensão proporcionam além de agilidade e maior segurança, redução de riscos ocupacionais como as lesões por esforço repetitivo e os distúrbios osteomusculares relacionados ao trabalho (LER e DORT).

Figura 1 - Ficha Clínica 
Figura 2 - Sistema Reciproc ${ }^{\circledR}$ e Sistema Race ${ }^{\circledR}$

Figura 3 - Instrumentos da técnica manual (limas, gattes glidden e espaçadores)

Figura 4 - Localizador apical e Motor endodôntico

Figura 5 - Sistema digital de Radiografia
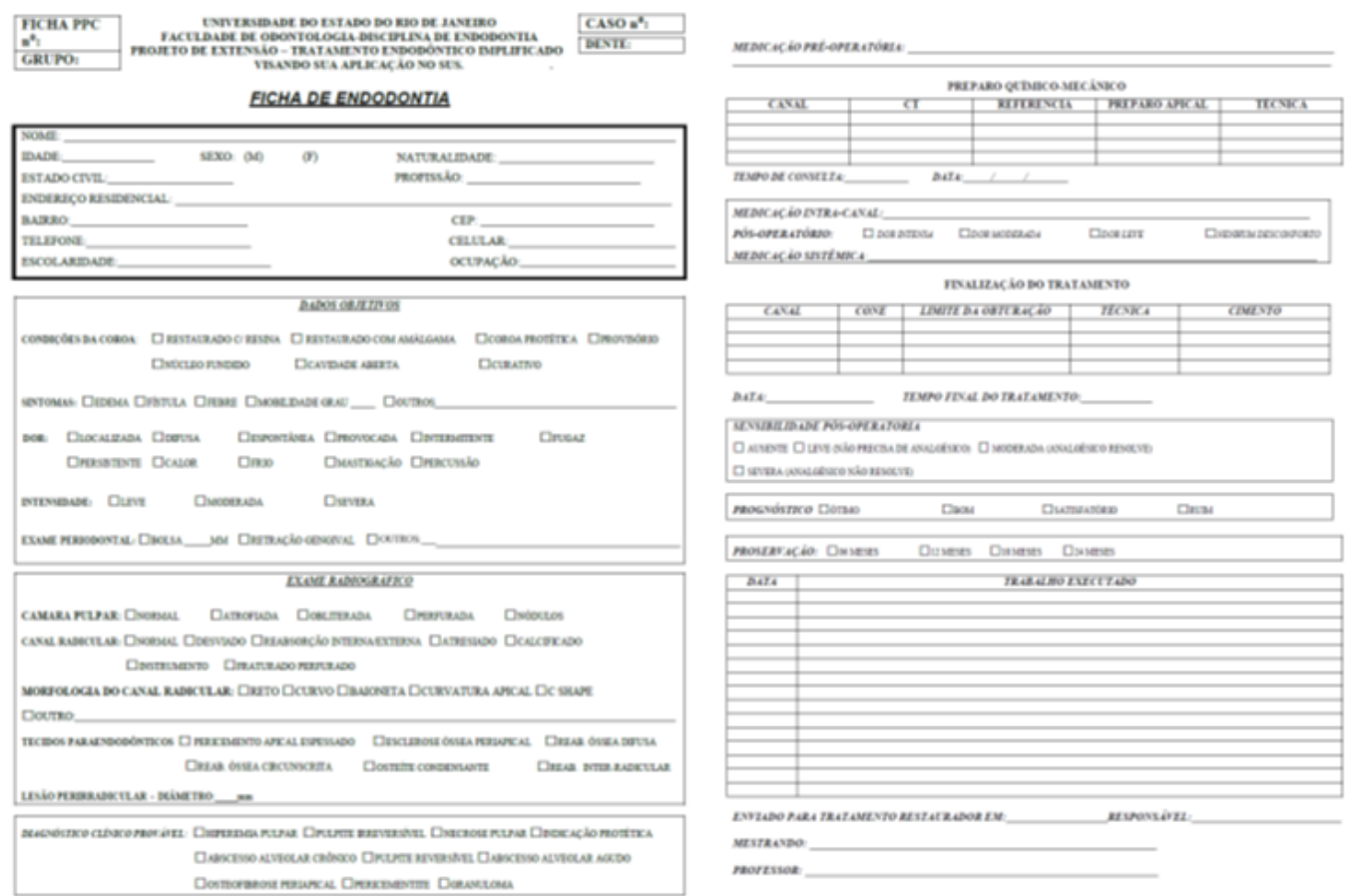

Figura 1 - Ficha Clínica

Fonte: Elaborada pelos autores, 2019
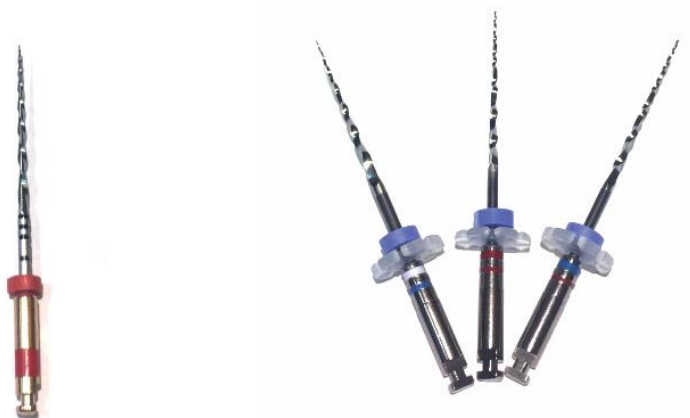

\section{A}


Figura 2 - Sistema Reciproc ${ }^{\circledR}$ e Sistema Race ${ }^{\circledR}$
A) Lima Reciproc ${ }^{\circledR}$ R25.
B) Sistema Race®.

Fonte: Elaborada pelos autores, 2019

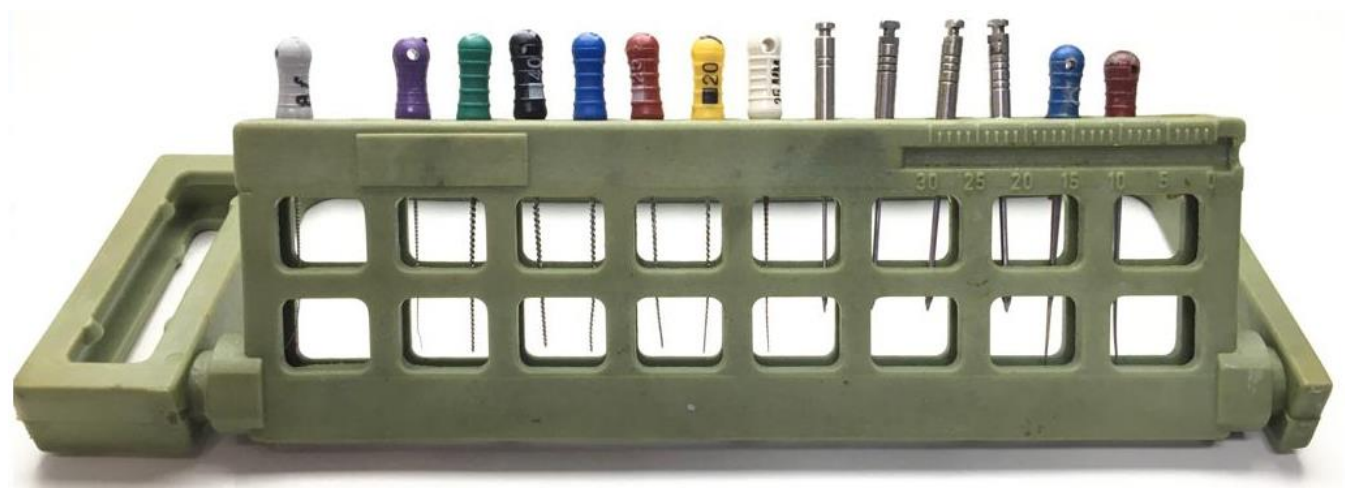

Figura 3 - Instrumentos da técnica manual (limas, gattes glidden e espaçadores)

Fonte: Elaborada pelos autores, 2020
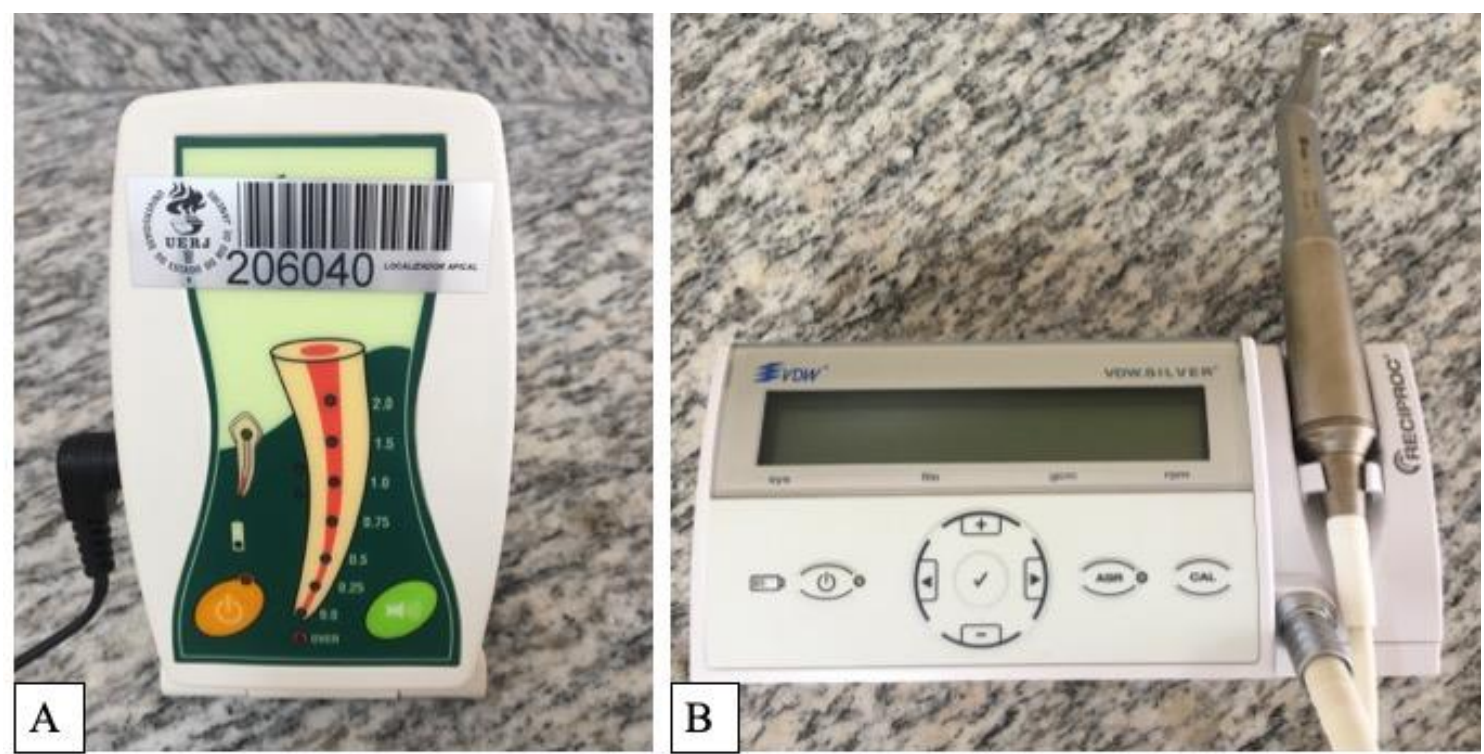
Figura 4 - Localizador apical e Motor endodôntico

A) Localizador apical Novapex® (Forum Technologies, Rishon Le-Zion, Israel).

B) Motor endodôntico da marca VDW Silver Reciproc ${ }^{\circledR}$ (VDW, Munique, Alemanha).

Fonte: Elaborada pelos autores, 2019.
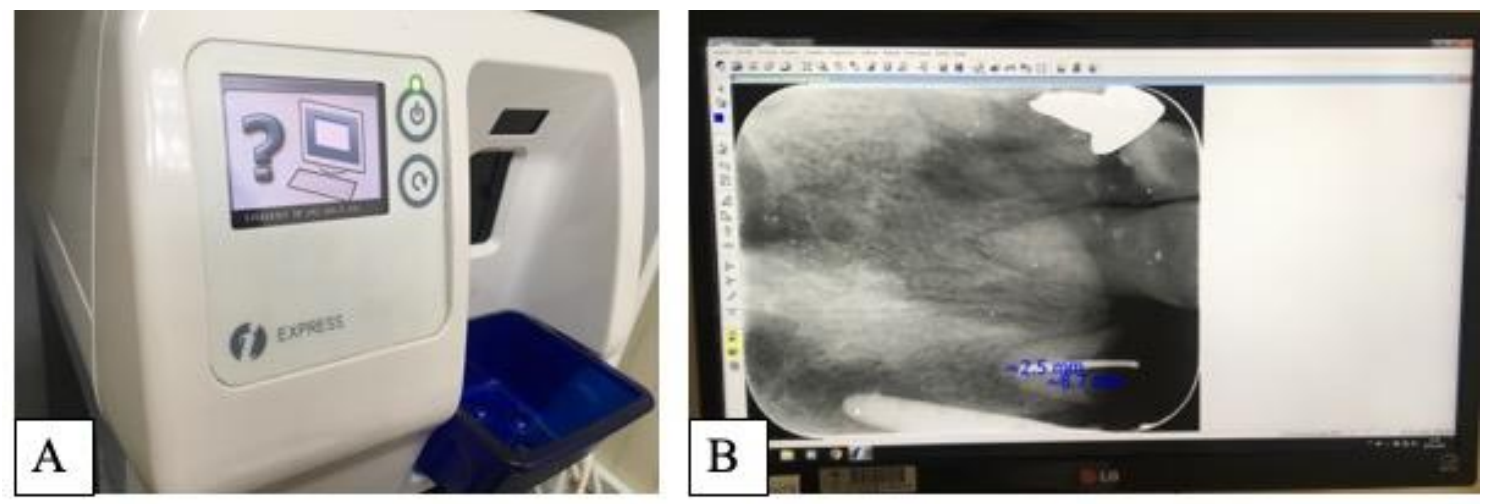

Figura 5 - Sistema digital de Radiografia

A) Sistema digital EXPRESS $®$ de radiografia com placa de fósforo (Kavo, Santa Catarina, Brasil).

B) Radiografia digital visualizada diretamente na tela do computador.

Fonte: Elaborada pelos autores, 2019

\section{Considerações Finais}

Desde a implementação das novas diretrizes curriculares (resolução n³ do CNE/CSE, de 2002), os cursos de graduação vêm abordando uma formação mais humanista (saudável, cordial e de respeito mútuo), crítica e reflexiva, para a atuação em todos os níveis de atenção em saúde. ${ }^{24}$ Neste contexto, a extensão universitária é uma importante ferramenta de interação entre o estudante universitário e a comunidade, sendo ambos beneficiados. A experiência extramuros do 
acadêmico em odontologia garante novos conhecimentos e habilidades importantes para a futura prática clínica, tornando-o mais ajustado às exigências do Sistema Único de Saúde, além de atuar diretamente nos problemas da comunidade. ${ }^{25,26}$

Ao longo desses cinco anos de existência, o projeto de extensão "Tratamento endodôntico simplificado visando sua aplicação no SUS" tem contribuído para a formação de recursos humanos (no âmbito da graduação e pós-graduação), para a produção de conhecimento científico e para a garantia de um tratamento mais especializado com maior eficiência para a comunidade.

Através de resultados parciais da pesquisa desenvolvida no projeto, foi possível verificar que a inclusão da instrumentação automatizada e outras tecnologias durante o tratamento endodôntico, permite efetiva redução no tempo de atendimento e consequentemente contribui para a diminuição da demanda reprimida de pacientes encaminhados para o Setor de Endodontia. Dessa forma, o investimento em tecnologias parece ser uma alternativa viável ao setor público que pretende oferecer serviços que promovam a saúde bucal baseada nos princípios do SUS da universalidade, a integralidade e a equidade, além de promover maior ergonomia e redução de riscos ocupacionais ao cirurgião-dentista.

\section{Agradecimentos}

A todos os colaboradores, estudantes universitários e pacientes que contribuíram para que o projeto "Tratamento endodôntico simplificado visando sua aplicação no SUS" se desenvolvesse e alcançasse seus objetivos.

\section{Referências}

1. BRASIL. Ministério da Educação. Secretaria de Educação Superior. Extensão Universitária: Organização e Sistematização. Política Nacional de Extensão. Universidade Federal de Minas Gerais. PROEX. COOPMED Editora, 2007. 
2. FIGUEIREDO N.; GOES, P.S.A.D. Construção da atenção secundária em saúde bucal: um estudo sobre os Centros de Especialidades Odontológicas em Pernambuco, Brasil. Cadernos de Saúde Pública, v. 25, p. 259-267, 2009.

3. PIVETTA, H.; FOllETO, M. Ensino, pesquisa e extensão universitária: em busca de uma integração efetiva. Linhas Críticas, v. 16, n. 31, p. 377-390, 2010.

4. SALIBA, N.A.; NAYME J.G.R.; MOIMAZ S.A.S.; CECILIO L.P.P.; GARBIN C.A.S. Organização da demanda de um Centro de Especialidades Odontológicas. Revista de Odontologia da UNESP, p. 317-323, 2013.

5. ____-_. Conselho Nacional de Secretários da Saúde. Conass. Atenção Primária e Promoção da Saúde: coleção para Entender a Gestão do SUS. Brasìlia: CONASS, 2011. 197 p, livro 3.

6. SERRA, C.G.; RODRIGUES, P.H.A. Avaliação da referência e contrarreferência no Programa Saúde da Família na Região Metropolitana do Rio de Janeiro (RJ, Brasil). Ciência \& Saúde Coletiva, v. 15, p. 3579-3586, 2010.

7. FRAZÃO, P.; NARVAI, P. C. Construção da atenção secundária em saúde bucal: um estudo sobre os Centros de Especialidades Odontológicas em Pernambuco, Brasil. Cadernos de Saúde Pública, v. 25, p. 259-267, 2009

8. CHAVES S.C.L. Atenção à saúde bucal e a descentralização da saúde no Brasil: estudo de dois casos exemplares no Estado da Bahia. Cadernos de Saúde Pública, v. 23, p. 1119-1131, 2007.

9. LIMA, A.C.S.D.; CABRAL, E.D.; VASCONCELOS, M.M.V.B. Satisfação dos usuários assistidos nos Centros de Especialidades Odontológicas do município do Recife, Pernambuco, Brasil. Cadernos de Saúde Pública, v. 26, p. 991-1002, 2010.

10. SALIBA, N.A; NAYME, J. G. R., MOIMAZ, S. A. S. et al. Organização da demanda de um Centro de Especialidades Odontológicas. Revista de Odontologia da UNESP, v. 42, n. 5, p. 317-323, 2013. 
11. ANTUNeS, J. L. F; NARVAI, P. C. Políticas de saúde bucal no Brasil e seu impacto sobre as desigualdades em saúde. Revista de Saúde Pública, v. 44, p. 360 $365,2010$.

12. MOREIRA, R. D. S.; NICO, L. S.; TOMITA, N. E.; RUIZ, T. A saúde bucal do idoso brasileiro: revisão sistemática sobre o quadro epidemiológico e acesso aos serviços de saúde bucal. Cadernos de Saúde Pública, v. 21, p. 1665-1675, 2005.

13. GAVINI G.; SANTOS M.; CALDEIRA C. L.; MACHADO, M. E. D. L.; FREIRE, L. G.; IGLECIAS, E. F.; CANDEIRO, G. T. D. M. Nickel-titanium instruments in endodontics: a concise review of the state of the art. Brazilian Oral Research, v. 32, 2018.

14. GOLDBERG F.; FRAJLICH S.; KUTTLER S.; MANZUR; BRISEÑOMARROQUÍN, B. The evaluation of four electronic apex locators in teeth with simulated horizontal oblique root fractures. Journal of Endodontics, v. 34, n. 12, p. 1497-1499, 2008.

15. BORG, E.; GRÖNDAHL, H. G. On the Dynamic Range of Different X-ray Photon Detectors in Intra-Oral Radiography. A Comparison of Image Quality in Film, Charge-Coupled Device and Storage Phosphor Systems. Dentomaxillofac Radiol, v. 25, n. 2, p. 82- 88, 1996.

16. PALEKER, F.; VAN DER VYVER, P. J. Glide path enlargement of mandibular molar canals by using K-Files, the ProGlider File, and G-Files: a comparative study of the preparation times. Journal of Endodontics, v. 43, n. 4, p. 609-612, 2017.

17. SUN, C.; SUN, J.; TAN, M.; HU, B.; GAO, X.; SONG, J. after root canal treatment with different instruments: A systematic review and meta-analysis. Oral diseases, v. 24, n. 6, p. 908-919, 2018.

18. SIQUEIRA JR, J. F. Microbial causes of endodontic flare-ups. International Endodontic Journal, v. 36, n. 7, p. 453-463, 2003.

19. AHLQUIST, M.; HENNINGSSON, O.; HULTENBY, K.; OHLIN, J. The effectiveness of manual and rotary techniques in the cleaning of root canals: a scanning electron microscopy study. International Endodontic Journal, v. 34, n. 7, p. 533-537, 2001. 
20. AHN, S. Y.; KIM, H. C.; KIM, E. Kinematic effects of nickel-titanium instruments with reciprocating or continuous rotation motion: a systematic review of in vitro studies. Journal of Endodontics, v. 42, n. 7, p. 1009-1017, 2016.

21. BÜRKLEIN, S.; SCHÄFER, E. Apically extruded debris with reciprocating singlefile and full-sequence rotary instrumentation systems. Journal of Endodontics, v. 38, n. 6, p. 850-852, 2012.

22. KRITHIKADATTA, J.; SEKAR, V.; SUDHARSAN, P.; VELUMURUGAN, $\mathrm{N}$. Influence of three $\mathrm{Ni}-\mathrm{Ti}$ cleaning and shaping files on postinstrumentation endodontic pain: A triple-blinded, randomized, controlled trial. Journal of Conservative Dentistry: JCD, v. 19, n. 4, p. 311, 2016.

23. GUELZOW A.; STAMM O.; MARTUS P.; KIELBASSA A. M. Comparative study of six rotary nickel-titanium systems and hand instrumentation for root canal preparation. International Endodontic Journal, v. 38, n. 10, p. 743-752, 2005.

24. SONNTAG D.; DELSCHEN S.; STACHNISS V. Pain after root canal treatment with different instruments: A systematic review and meta-analysis. Oral diseases, v. 24, n. 6, p. 908-919, 2018.

25. OLIVEIRA, J. K.; VIEIRA, L. E.; LIMEIRA, M. S.; ARAÚJO, M. G.; OLIVEIRA, D. H.; CHACON, L. D.; RODRIGUES, R. Q. Práticas educativas em saúde bucal direcionadas aos usuários do restaurante popular da cidade de Patos-PB. Revista Brasileira de Extensão Universitária, v. 8, n. 2, p. 67-74, 2017.

26. WARMLING, C.M.; ROSSONI, E.; HUGO, F.N.; TOASSIi, R. F. C.; de LEMOS, V. A.; de SILAVUTZKI, S. M. B.; da ROSA, A. R. Estágios curriculares no SUS: experiências da Faculdade de Odontologia da UFRGS. Revista da ABENO, v. 11, n. 2, p. 63-70, 2011.

27. BISCARDE, D. G. D. S.; PEREIRA-SANTOS, M.; SILVA, L. B. Formação em saúde, extensão universitária e Sistema Único de Saúde (SUS): conexões necessárias entre conhecimento e intervenção centradas na realidade e 
Novas Tecnologias em Endodontia e seu uso no atendimento de pacientes de uma unidade de saúde pública: Cinco anos de atividade extensionista

repercussões no processo formativo. Interface-Comunicação, Saúde, Educação, v. 18, p. 177-186, 2014. 